\title{
Synthesis and Characterization of Polyesteramide Hot Melt Adhesive from Low Purity Dimer Acid, Ethylenediamine, and Ethanolamine
}

\author{
Pravin G. Kadam, ${ }^{1}$ Parth Vaidya, ${ }^{2}$ and Shashank T. Mhaske ${ }^{1}$ \\ ${ }^{1}$ Department of Polymer and Surface Engineering, Institute of Chemical Technology, Matunga, Mumbai, Maharashtra 400 019, India \\ ${ }^{2}$ Department of Oils, Oleochemicals and Surfactants Technology, Institute of Chemical Technology, Matunga, Mumbai, \\ Maharashtra 400 019, India \\ Correspondence should be addressed to Shashank T. Mhaske; stmhaske@gmail.com
}

Received 7 December 2013; Revised 3 March 2014; Accepted 11 March 2014; Published 7 April 2014

Academic Editor: Beng T. Poh

Copyright (c) 2014 Pravin G. Kadam et al. This is an open access article distributed under the Creative Commons Attribution License, which permits unrestricted use, distribution, and reproduction in any medium, provided the original work is properly cited.

\begin{abstract}
Polyesteramide hot melt adhesive (HMA) was synthesized using low purity dimer acid (composition: 3\% linoleic acid, 75\% dimer acid, and 22\% trimer acid), ethanolamine, and ethylenediamine. Ethanolamine was added as a partial replacement (10, 20, and 30\%) of ethylenediamine. Prepared HMAs were characterized for acid value, amine value, hydroxyl value, Fourier transform infrared spectroscopy, mechanical (tensile strength, percentage strain at brea, and shore D hardness), thermal (glass transition temperature, melting temperature, enthalpy of melting, crystallization temperature, and enthalpy of crystallization), rheological (viscosity versus shear rate and viscosity versus time), and adhesion properties (T-peel strength and lap shear strength). Replacement of ethylenediamine by ethanolamine replaced certain amide linkages by ester linkages, decreasing the intermolecular hydrogen bonding, leading to decrease in the crystallinity of the material, and thus the mechanical, thermal, adhesion, and rheological properties. However, HMAs prepared using ethanolamine will have better low temperature flexibility due to low glass transition temperature and better adhesion process due to the lower viscosity.
\end{abstract}

\section{Introduction}

Hot melt adhesives (HMAs) are solid adhesives which when heated are converted to a molten liquid state for application to substrates whereas on cooling quickly, they set up the bond [1]. The substrates must be joined immediately on application of the HMA [2]. When hardened, HMA can have various degrees of tackiness depending on the formulation. Conventional HMAs cool to harden and do not chemically crosslink. Such systems have an open time from few seconds to few minutes [1].

Materials that are primarily used as HMAs include ethylene and vinyl acetate copolymers (EVA), polyvinyl acetates $(\mathrm{PVAc})$, polyethylene (PE), amorphous polypropylene (PP), block copolymers (styrene butadiene rubber, SBR), polyamides (PA), and polyester (PEster). HMAs are solid at temperatures below $80^{\circ} \mathrm{C}$. Typical application temperatures are $150-200^{\circ} \mathrm{C}[2]$.
The most important advantage of HMA is the ability of being able to preapply, for example, as powder or adhesive spheres, in melt liquid form, as dispersion or as an adhesive foil. The joining procedure does not have to take place directly after applying the adhesive to the substrate; this can happen at any time later on [3]. The market for HMA is mainly in the manufacture of durable goods. Examples are shoe assembly, kitchen and bathroom cabinets, telecommunication cable repair sleeves, and window assembly [4].

First PEA based HMA was reported by Martins and Ashley in 1972. They prepared PEA HMA from dimer acid, sebacic acid, hexamethylenediamine, and ethylene glycol and had bond strength of about 13.8 MPa. PEA synthesized from dimer acid acids, diols, and diamines had low crystallinity and wide range of melting temperatures, which make them particularly suitable to be used as a HMA [5]. Krieger et al. synthesized hot melt pressure sensitive adhesive by 
reacting dimer acid with ethanolamine and also dimer acid with mix of ethylenediamine and dimethyl terephthalate. Prepared adhesives were found to have adhesion strength of 0.34 and $0.47 \mathrm{MPa}$, respectively, and were used for sticking polystyrene labels to glass [6]. Martins and Donermeyer prepared spherical metal particles filled PEA HMA for cavity filling applications. PEA consisted of 60 percent by weight of polyethylene terephthalate and 40 percent by weight of polyamide prepared from dimer acid and hexamethylenediamine with a minor amount of antioxidant. This PEA was filled with spherical alumina, iron, mild steel, stainless steel, and zinc particles of varied particle size and concentration $[7,8]$. Veazey investigated the PEA HMA for joining vinyl based substrates. He synthesized PEA HMA by reacting 0.201 equivalents (eq.) of dimer acid with 1.82 eq. of sebacic acid, 1.95 eq. ethylenediamine, 0.21 eq. 1,4cyclohexane dimethanol, and 0.06 eq. stearic acid. Dimer acid used was of $95.6 \%$ purity. Viscosity of the polymer was determined to be $59000 \mathrm{cps}$, softening point of $162^{\circ} \mathrm{C}$, and had T-peel strength of 7.6 MPa [9]. Frihart and Veazey prepared PEA HMA by reacting 0.92 eq. dimer acid with 0.4 eq. sebacic acid, 0.41 eq. ethylenediamine, 0.97 eq. $\mathrm{N}$-(2-hydroxyethyl) piperazine, and 0.02 eq. stearic acid. Prepared HMA had viscosity of $15,500 \mathrm{cps}$ at $195^{\circ} \mathrm{C}$ and a softening point of $115^{\circ} \mathrm{C}$. It had improved adhesion strength at low temperature $\left(3.6 \mathrm{MPa}\right.$ at $\left.0^{\circ} \mathrm{C}\right)$ compared to ambient temperature $\left(1.9 \mathrm{MPa}\right.$ at $\left.22^{\circ} \mathrm{C}\right)$ for vinyl based substrates [10]. They synthesized many such PEA HMAs for vinyl substrates $[11,12]$. Hayes et al. synthesized PEA HMA from dimer acid (70\%), sebacic acid (30\%), ethylenediamine (45\%), and 1,6hexamethylenediamine (55\%). Prepared HMA was found to have viscosity of $6,000 \mathrm{cps}$ at $195^{\circ} \mathrm{C}$ and lap shear strength of 5.7 MPa [13]. Swan and Hansen developed PEA based HMA suitable for bonding polyester and polycarbonate substrates by reacting dimer acid, adipic acid, hexamethylenediamine, and ethylene glycol in varied proportion [14]. Chen et al. synthesized PEA of different viscosities using dimer acid, sebacic acid, ethylenediamine, and diethylene glycolamine. They determined improvement in mechanical and adhesion properties with increase in inherent viscosity of the PEA [15]. They also investigated the effect of PEA addition on the properties of EVA based HMA and found that PEA/EVA blend composition containing about 20\% PEA showed the highest miscibility and the best possible improvement in adhesion properties [16]. Broos et al. synthesized biodegradable PEA HMA using $1.2 \mathrm{~kg}$ ethylenediamine, $4.56 \mathrm{~kg} \varepsilon$-caprolactone, $2.62 \mathrm{~kg}$ dimethyl adipate, and $1.35 \mathrm{~kg}$ 1,4-butanediol [17]. Appleman et al. synthesized PEA HMAs using $258 \mathrm{~g}$ dimer acid, $44.4 \mathrm{~g}$ ethylenediamine, and $24.6 \mathrm{~g}$ dimer diol. Prepared HMA had softening point of $95^{\circ} \mathrm{C}$, glass transition temperature of $-46^{\circ} \mathrm{C}$, and lap shear strength of $28 \mathrm{~J} / \mathrm{mm}^{2}$ [18]. Kadam et al. investigated the effect of addition of modified Jatropha seed oil on the adhesion properties of hot melt adhesive synthesized using dimer acid and ethylenediamine [19].

This study aims to synthesize PEA based HMA from lower purity dimer acid, ethanolamine, and ethylenediamine. Prepared HMAs were characterized for acid value, amine value, hydroxyl value, Fourier transform infrared
TABLE 1: Molecular weight of the reactants used in preparing PEA HMA.

\begin{tabular}{lcc}
\hline Serial number & Reactant & Molecular weight $(\mathrm{g} / \mathrm{mol})$ \\
\hline 1. & Trimer acid & 840 \\
2. & Dimer acid & 560 \\
3. & Linoleic acid & 280 \\
4. & Ethylenediamine & 60 \\
5. & Ethanolamine & 61 \\
\hline
\end{tabular}

spectroscopy, mechanical [tensile strength (TS), percentage elongation at break (PEB), shore D hardness (SD)], thermal [melting temperature $(\mathrm{Tm})$, enthalpy of melting $(\mathrm{Hm})$, crystallization temperature $(\mathrm{Tc})$, enthalpy of crystallization $(\mathrm{Hc})$ and glass transition temperature $(\mathrm{Tg})$ ], rheological [viscosity versus shear rate and viscosity versus time], and adhesion [lap shear strength (LSS) and T-peel strength (TPS)] properties.

\section{Experimental}

2.1. Materials. Lower purity dimer acid [Pripol 1017; composition: $\sim 3 \%$ linoleic acid, $\sim 75 \%$ dimer acid, and $\sim 22 \%$ trimer acid] with an acid value of $198 \mathrm{mg} \mathrm{KOH} / \mathrm{g}$ was obtained from Croda Chemicals, Mumbai, India. Ankita Chemicals, Mumbai, India, supplied Ethylenediamine. Ethanolamine was procured from S.D. Fine Chemicals Pvt. Ltd., Mumbai, India. All chemical compounds were used as received, without any modification or purification.

\subsection{Preparation of Polyesteramide Hot Melt Adhesive.} Figure 1 shows the molecular structure of the reactants used in preparing PEA HMA, whereas their molecular weights are listed in Table 1.

PEA HMAs were prepared by reacting lower purity dimer acid with EDA. In order to understand the effect of EOA addition on the properties of the HMA, some part of EDA was replaced by EOA, on molar basis. Accordingly, EDA was replaced by 10,20 , and $30 \%$ of EOA. Prepared formulations and their nomenclature are reported in Table 2. Below mentioned example illustrates the calculations involved in preparing PEA10.

Pripol 1017 (lower purity dimer acid) consists of 75\% dimer acid, 22\% trimer acid, and 3\% linoleic acid. Thus, $200 \mathrm{gm}$ of Pripol 1017 consisted of $150 \mathrm{~g}$ dimer acid, $44 \mathrm{~g}$ trimer acid, and $6 \mathrm{~g}$ linoleic acid; that is, it had 0.27 moles of dimer acid, 0.05 moles of trimer acid, and 0.02 moles of linoleic acid. Functionality of trimer acid is 3, while that of all EDA and EOA is 2. So, the number of moles of EDA and EOA required in equivalent to trimer acid are more and it would be required in the ratio $1: 1.5$ (trimer acid: EOA/EDA), so as to equalize the functionality of the compounds. Number of moles will be accordingly distributed between EDA and EOA. Indirectly, number of moles of trimer acid can be considered as 1.5 times of its original quantity, so that the calculation and distribution among EDA and EOA becomes easy, whereas, the functionality of linoleic acid is 1 . Thus, the number of moles of linoleic acid will be considered as 


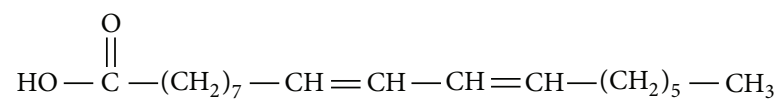

(a)

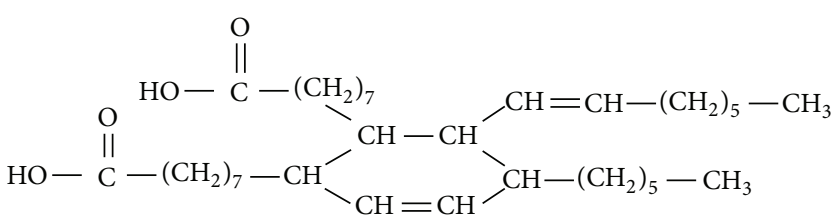

(b)

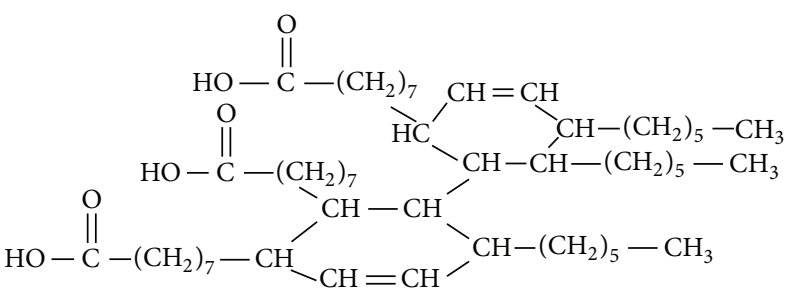

(c)

$$
\mathrm{H}_{2} \mathrm{~N}-\mathrm{CH}_{2}-\mathrm{CH}_{2}-\mathrm{NH}_{2} \quad \mathrm{HO}-\mathrm{CH}_{2}-\mathrm{CH}_{2}-\mathrm{NH}_{2}
$$

(d)

(e)

Figure 1: Molecular structure of the reactants used in the preparation of the PEA HMAs.

TABLE 2: Prepared PEA HMA formulations with their nomenclature.

\begin{tabular}{lcccr}
\hline Serial number & Nomenclature & $\begin{array}{c}\text { Pripol } 1017 \text { mole } \\
(\mathrm{g})\end{array}$ & $\begin{array}{c}\text { Ethylenediamine (EDA) } \\
\text { mole }(\mathrm{g})\end{array}$ & $\begin{array}{c}\text { Ethanolamine (EOA) } \\
\text { mole }(\mathrm{g})\end{array}$ \\
\hline 1. & PEA0 & $0.36(200)$ & $0.36(21.60)$ & - \\
2. & PEA10 & $0.36(200)$ & $0.32(19.20)$ & $0.04(2.44)$ \\
3. & PEA20 & $0.36(200)$ & $0.28(16.80)$ & $0.08(4.88)$ \\
4. & PEA30 & $0.36(200)$ & $0.24(14.40)$ & $0.12(7.32)$ \\
\hline
\end{tabular}

0.5 times of its original quantity. Accordingly, Pripol 1017 now consisted of 0.27 moles of dimer acid, 0.08 moles of trimer acid, and 0.01 moles of linoleic acid. Total moles of acid present in Pripol 1017 are 0.36. Pripol 1017 was reacted with EDA and EOA in 1:1 molar ratio (EDA and EOA considered together), so as to prepare the HMA of the highest possible molecular weight. 0.36 moles of Pripol 1017 had been reacted with 0.36 moles of EDA and EOA (both combined). This formulation relates to PEA HMA preparation in which $10 \%$ of EDA is replaced by EOA. Accordingly, 0.36 moles will be divided as 0.32 moles (90\%) of EDA and 0.04 moles (10\%) of EOA. Thus, $200 \mathrm{~g}$ Pripol 1017 (low purity dimer acid) is reacted with $19.20 \mathrm{~g}$ EDA and $2.44 \mathrm{~g}$ EOA. PEA0 was prepared without any addition of EOA.

To a four necked $500 \mathrm{~mL}$ reactor, equipped with stirring system, condenser, nitrogen gas inlet, and temperature controller, $200 \mathrm{~g}$ (0.36 moles lower purity dimer acid) Pripol 1017, $19.20 \mathrm{~g}$ (0.32 moles) EDA, and $2.44 \mathrm{~g}$ (0.04 moles) EOA were added. The reactants were heated gradually to $250^{\circ} \mathrm{C}$ with stirring at $1000 \mathrm{rpm}$, under nitrogen atmosphere. Acid and amine value of the reacting mixture was measured after every $30 \mathrm{~min}$ in order to determine the progress of the reaction. The procedure for measuring the acid and amine value of the reaction mixture is mentioned below. This temperature was maintained till the acid and amine value for the system decreased below 5 , requiring about $8 \mathrm{~h}$, thus preparing PEA10. PEA0, PEA20, and PEA30 were also synthesized according to the above typical synthesis methodology. PEA0 did not contain EOA. PEAs were prepared by melt polycondensation method without the use of solvent or catalyst. None of the reactants were expected to remain unreacted in the mixture. So, no purification step was thought to be necessary for the final products.

\section{Characterization and Testing}

3.1. Acid Value. $50 \mathrm{~mL}$ benzene-alcohol mixture was added to dissolve $2 \mathrm{~g}$ sample in $125 \mathrm{~mL}$ Erlenmeyer flask, warming in water bath if necessary. Solution was cooled (if necessary) and was added with a few drops of phenolphathalein indicator. The solution was titrated against $0.5 \mathrm{~N}$ alcoholic potassium hydroxide solution to a faint pink color which remained for 30 seconds or more. The calculation of acid value is shown below:

Acid Value

$$
=\frac{(56.1 \times \text { Volume of alc. } \mathrm{KOH} \times \text { Normality of alc. } \mathrm{KOH})}{(\text { Weight of sample })} .
$$

3.2. Amine Value. $50 \mathrm{~mL}$ isopropyl alcohol was added to dissolve $2 \mathrm{~g}$ sample in $125 \mathrm{~mL}$ Erlenmeyer flask, warming on a heat source if necessary. Solution was cooled (if necessary) was added with a few drops of bromocresol green indicator. The solution was titrated against $0.5 \mathrm{~N}$ standardized hydrochloric acid $(\mathrm{HCl})$ to a yellowish green color which 
remained for 30 seconds or more. The calculation of amine value is shown in below:

Amine Value

$$
=\frac{(56.1 \times \text { Volume of } \mathrm{HCl} \times \text { Normality of } \mathrm{HCl})}{(\text { Weight of sample })} .
$$

3.3. Hydroxyl Value. $5 \mathrm{~g}$ sample was accurately weighed in a $250 \mathrm{~mL}$ Erlenmeyer flask, whereas other Erlenmeyer flasks had no added sample, to obtain blank reading. Both the flasks were then added with $20 \mathrm{~mL}$ of acetic anhydride-pyridine reagent (1:4 volume ratio) and attached with air condenser. Flasks were then immersed up to the liquid level in an oil bath maintained at $95-100^{\circ} \mathrm{C}$. Flasks were swirled vigorously to bring about complete dissolution of the sample in the reagent. Flasks were heated continuously for 1 hour to insure uniform reaction. Flasks were cooled to room temperature. Then, $10 \mathrm{~mL}$ water was added to the flasks through the air condenser. Flasks were again heated on oil bath for $15 \mathrm{~min}$ to hydrolyze the excess acetic anhydride reagent. Flasks were then cooled to room temperature and added with $25 \mathrm{~mL}$ neutralized alcohol (half through the condenser, which was removed afterwards). Prepared mixture was stirred rigorously. Mixture was made ready for titration on addition of $1 \mathrm{~mL}$ phenolphthalein indicator and was titrated against $0.5 \mathrm{~N}$ alcoholic potassium hydroxide to a faint pink color, which remains for 30 seconds or more. The calculation of hydroxyl value is shown follows:

$$
\begin{aligned}
& \text { Hydroxyl Value } \\
& \begin{aligned}
= & {[56.1 \times \text { Normality of alc. } \mathrm{KOH}} \\
& \times(\mathrm{mL} \text { KOH blank }-\mathrm{mL} \mathrm{KOH} \text { sample })] \\
& \times[\text { Weight of Sample }]^{-1} \\
& - \text { Acid Value. }
\end{aligned}
\end{aligned}
$$

3.4. FTIR. The FTIR spectra were recorded with a PerkinElmer, Spectrum GX equipment, USA. 1-2 wt\% solution of HMA was dissolved in chloroform and was scanned with a resolution of $2 \mathrm{~cm}^{-1}$ in the scan range of $450-4000 \mathrm{~cm}^{-1}$. FTIR of pure solvent was run prior to running the FTIR of the samples, to use its peaks as baseline, to automatically subtract it from the samples peak.

3.5. Mechanical Properties. Tensile strength of compression molded sheet was measured in accordance with ASTM D638 on Universal Testing Machine (LR-50K, Lloyds Instrument, UK). Crosshead speed, for testing, was maintained at $50 \mathrm{~mm} / \mathrm{min}$. Shore D hardness was determined in accordance with ASTM D2240 on Shore "D" Hardness Tester (International Equipments, Mumbai, India). Both the tests were performed at ambient conditions of $25^{\circ} \mathrm{C}$ and $75 \%$ relative humidity.

3.6. Thermal Properties. Differential scanning calorimetry (Q100 DSC, TA Instruments Ltd., India) was used to investigate the crystallization and melting behavior of the HMA. Two consecutive scans were obtained to minimize the influence of possible residual stresses in the material due to any specific thermal history. A scanning rate of the $10^{\circ} \mathrm{C} /$ minute was used for both the exo- and endothermal cycle with the nitrogen purge at $50 \mathrm{~mL} / \mathrm{min}$. The glass transition temperature (Tg) and enthalpy of melting ( $\mathrm{Hm})$ were determined from the second heating scan, while the enthalpy of crystallization ( $\mathrm{Hc}$ ) was determined from first cooling scan. The area under the melting or crystallization peak represents the amount of energy required to melt the polymer (Hf) and the amount of energy released during crystallization $(\mathrm{Hc})$, respectively. The peak area calculation is used with the limits of the calculation on the flat portion of the baseline before and after melting or crystallization peak. Peak temperatures were noted as the melting (Tm) and crystallization (Tc) temperatures.

3.7. Rheological Properties. The viscosity versus shear rate was determined at the temperature of $200^{\circ} \mathrm{C}$ up to maximum shear rate of $100 \mathrm{~s}^{-1}$, whereas viscosity versus time was determined at the temperature of $200^{\circ} \mathrm{C}$ at a constant shear rate of $1 \mathrm{~s}^{-1}$ for 400 seconds using MCR101 Rheometer (Anton Paar, Germany).

3.8. Adhesion Properties. PEA adhesive films were also obtained by compression molding. The adhesive joint was then obtained by pressing the film between two pieces of surface treated aluminum adherent at a temperature of $2000^{\circ} \mathrm{C}$ and at a pressure of $30 \mathrm{KPa}$ for $20 \mathrm{~min}$, followed by cooling at room temperature for $24 \mathrm{~h}$. Lap shear strength was determined according to ASTM D1002-72 at a crosshead speed of $1.3 \mathrm{~mm} / \mathrm{min}$. T-peel strength was measured in accordance with ASTM D1876 with a crosshead speed of $254 \mathrm{~mm} / \mathrm{min}$. Average thickness of adhesive layer in the lap and T-peel joints was kept within $0.035 \pm 10 \% \mathrm{~mm}$ (0.001 inch). Lap shear strength and T-Peel strength were all measured using a Universal Testing Machine (LR 50K, Lloyds Instruments, UK).

\section{Results and Discussion}

Due to the presence of trimer acid, chances exist of it forming a cross-linked structure. However, this is possible only with higher content of trimer acid. Trimer acid content in this reaction system is not high enough to produce crosslinking. Trimer acid is a bulky molecule. The chance of other bulky structures (oligomeric chains of the polyestermide/polyamide formed in the reaction) to come close to trimer acid and undergo reaction is less, due to the steric hindrance. So, instead of cross-linking, chances are higher for the formation of branched structure. This can be confirmed through rheological studies.

4.1. Acid Value, Amine Value and Hydroxyl Value. Acid, amine, and hydroxyl values obtained for the prepared PEA HMAs are listed in Table 3. It can be seen that acid value remained near constant, whereas, there is appreciable change 
TABLE 3: Acid, amine, and hydroxyl values obtained for the prepared PEA HMAs.

\begin{tabular}{lccc}
\hline Sample name & $\begin{array}{c}\text { Acid value } \\
(\mathrm{mg} \mathrm{KOH} / \mathrm{g})\end{array}$ & $\begin{array}{c}\text { Amine value } \\
(\mathrm{mg} \mathrm{KOH} / \mathrm{g})\end{array}$ & $\begin{array}{c}\text { Hydroxyl } \\
\text { value } \\
(\mathrm{mg} \mathrm{KOH} / \mathrm{g})\end{array}$ \\
\hline PEA0 & 4.3 & 4.1 & - \\
PEA10 & 4.7 & 3.8 & 2.7 \\
PEA20 & 4.0 & 3.2 & 3.4 \\
PEA30 & 4.8 & 2.5 & 4.7 \\
\hline
\end{tabular}

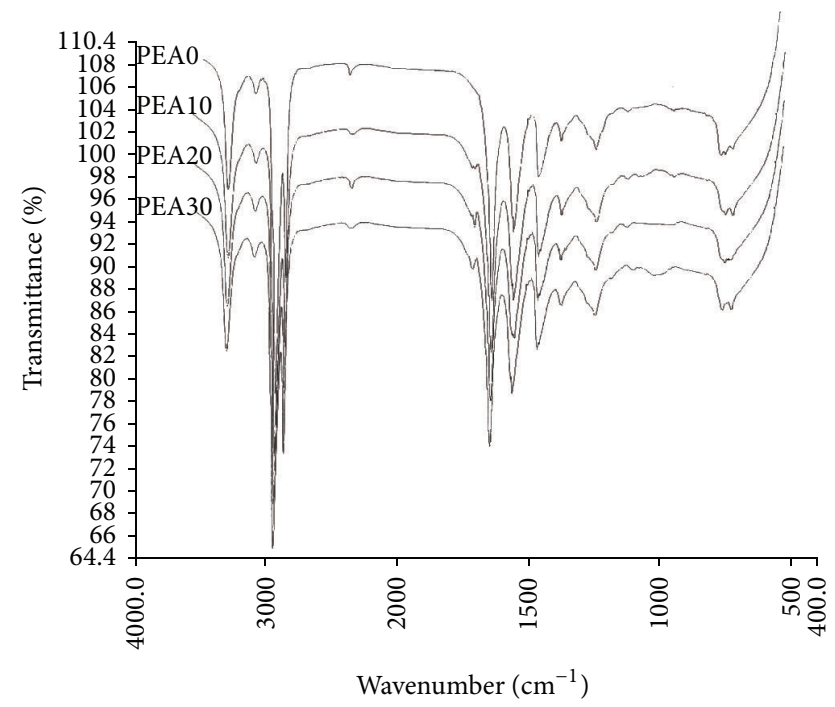

FIGURE 2: FTIR graphs obtained for the prepared PEA HMAs.

in the amine and hydroxyl values. Amine value decreased and hydroxyl value increased with increase in concentration of EOA in the formulation from PEA0 to PEA30.

In the formulations, part of EDA was replaced by EOA, replacing amine groups by hydroxyl groups. Accordingly, hydroxyl end groups, from PEA0 to PEA30, replaced more of amine end groups. This led to increase in hydroxyl value with subsequent decrease in amine value.

4.2. FTIR Analysis. FTIR transmittance curves obtained for the prepared PEAs are shown in Figure 2.

A small band of the amide I $\left(-\mathrm{NH}_{2}\right.$ in primary amides) groups appeared at about $3300 \mathrm{~cm}^{-1}$. Peaks at about $2900 \mathrm{~cm}^{-1}$ were due to asymmetric and symmetric stretching of $-\mathrm{CH}_{2}$. Small peak at around $1725 \mathrm{~cm}^{-1}$ corresponds to the ester linkage $(\mathrm{C}=\mathrm{O})$. Intensity of this peak increased with increase in concentration of EOA in the reactants, lower purity dimer acid and ethylenediamine, forming PEA. This indicates the increase in the number of ester linkages. The carbonyl peak $\left(-\mathrm{CONH}_{2}\right)$ of polyamide was at around $1640 \mathrm{~cm}^{-1}$ (steep peak). Amide II band $/ \mathrm{CH}_{2}$ asymmetric deformation was indicated by the peak of wavenumber $1560 \mathrm{~cm}^{-1}$. The peak at $1225 \mathrm{~cm}^{-1}$ corresponded to $\mathrm{C}-\mathrm{N}$ stretching vibration bond. Small peak at $750 \mathrm{~cm}^{-1}$ showed C-C deformation. FTIR analysis thus proves that compound formed was
TABLE 4: Mechanical properties obtained for the prepared PEA HMAs.

\begin{tabular}{lccc}
\hline Sample name & $\begin{array}{c}\text { Tensile } \\
\text { strength } \\
(\mathrm{MPa})\end{array}$ & $\begin{array}{c}\text { Percentage } \\
\text { strain } \\
\text { at break }(\%)\end{array}$ & Shore D hardness \\
\hline PEA0 & $8.3 \pm 0.7$ & $5.7 \pm 0.2$ & $47 \pm 3$ \\
PEA10 & $4.6 \pm 0.3$ & $13.6 \pm 0.7$ & $45 \pm 2$ \\
PEA20 & $2.4 \pm 0.2$ & $21.4 \pm 0.8$ & $40 \pm 3$ \\
PEA30 & $2.1 \pm 0.1$ & $26.1 \pm 0.9$ & $35 \pm 3$ \\
\hline
\end{tabular}

TABLE 5: Thermal properties obtained for the prepared PEA HMAs.

\begin{tabular}{lccccc}
\hline Sample name & $\mathrm{Tg}\left({ }^{\circ} \mathrm{C}\right)$ & $\mathrm{Tm}\left({ }^{\circ} \mathrm{C}\right)$ & $\mathrm{Hm}(\mathrm{J} / \mathrm{g})$ & $\mathrm{Tc}\left({ }^{\circ} \mathrm{C}\right)$ & $\mathrm{Hc}(\mathrm{J} / \mathrm{g})$ \\
\hline PEA0 & 4.26 & 97.6 & 16.77 & 83.83 & 8.17 \\
PEA10 & -1.92 & 81.99 & 15.18 & 74.14 & 5.70 \\
PEA20 & -6.29 & 72.87 & 15.05 & 70.09 & 3.87 \\
PEA30 & -6.58 & 72.58 & 9.68 & 64.52 & 2.40 \\
\hline
\end{tabular}

an amide. However, with the addition of EOA, polyamide from lower purity dimer acid and EDA got converted to PEA, proven through the presence of ester linkage peak in the FTIR curves.

4.3. Mechanical Properties. Mechanical properties - shore D hardness (SD), tensile strength (TS, MPa), and percentage strain at break (PEB, \%), obtained for the prepared PEA HMAs are sown in Table 4.

HMA prepared using lower purity dimer acid and EDA was found to have TS of $8.3 \pm 0.7 \mathrm{MPa}$ and PEB of 5.7 $\pm 0.2 \%$. It was determined that TS decreased, while PEB increased with increase in concentration of EOA (by partial replacement of EDA). Replacement of EDA with EOA led to the replacement of amide linkages by ester linkages. Due to the fact that there is no availability of hydrogen atom on the ester linkage, it was not able to form intermolecular hydrogen bonding as strong as that made by the amide linkage. Decrease in intermolecular forces of attraction led to decrease in the molecular packing per unit area, decreasing crystallinity of the HMA. Diminished crystallinity resulted in lower TS and SD. TS and SD decreased with increased concentration of EOA in the HMA. Decrease in crystallinity allowed better molecular movement, making it easier for them to pass over each other, increasing the elongational property. This was the reason for the increase in PEB with increase in concentration of EOA in the HMA.

4.4. Thermal Properties. Graphs of thermal properties like glass transition temperature ( $\mathrm{Tg}$ ), heating, and cooling curves are shown in Figures 3, 4, and 5 and the values of glass transition temperature $(\mathrm{Tg})$, melting temperature $(\mathrm{Tm})$, enthalpy of melting $(\mathrm{Hm})$, crystallization temperature (Tc), and enthalpy of crystallization $(\mathrm{Hc})$ are shown in Table 5.

HMA prepared using lower purity dimer acid and ethylenediamine was found to have $\mathrm{Tg}, \mathrm{Tm}, \mathrm{Hm}, \mathrm{Tc}$, and $\mathrm{Hc}$ of $4.26^{\circ} \mathrm{C}, 97.6^{\circ} \mathrm{C}, 16.77 \mathrm{~J} / \mathrm{g}, 83.83^{\circ} \mathrm{C}$, and $8.17 \mathrm{~J} / \mathrm{g}$, respectively. $\mathrm{Tg}, \mathrm{Tm}, \mathrm{Hm}, \mathrm{Tc}$, and Hc decreased with replacement of EDA 


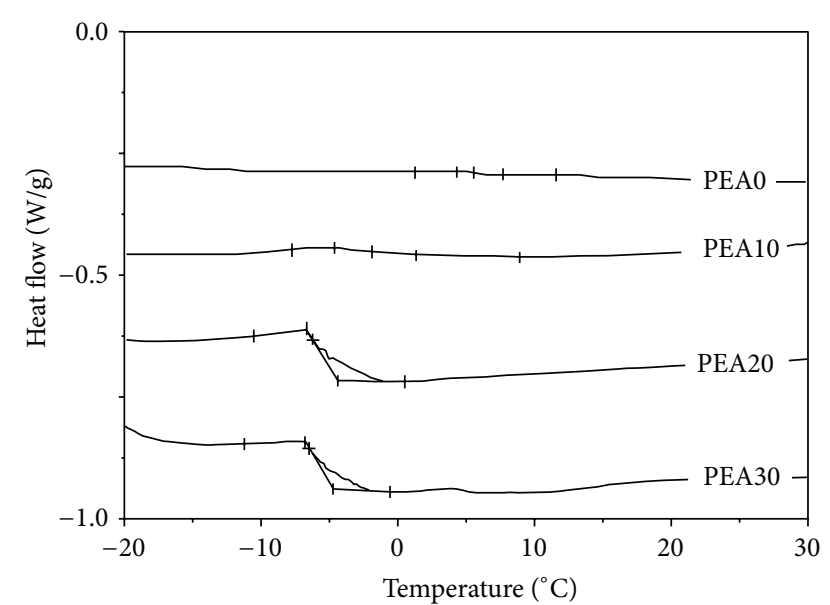

Figure 3: Glass transition curves obtained for the prepared PEA HMAs.

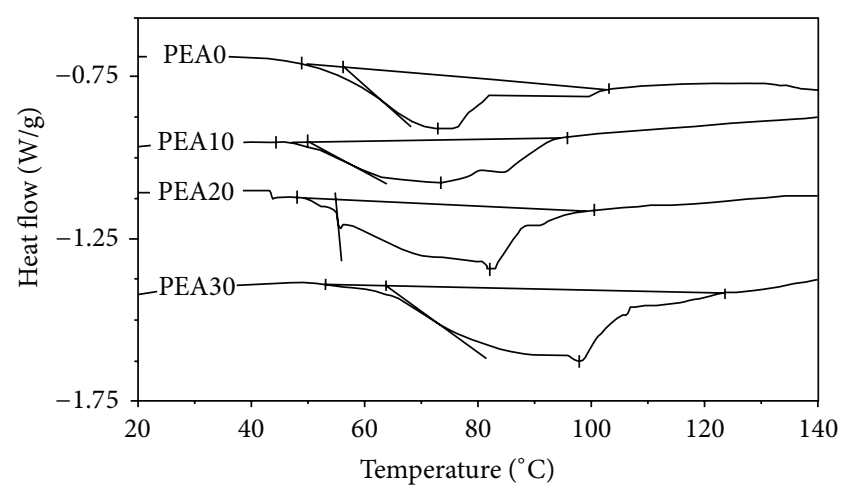

FIGURE 4: Heating curves obtained for the prepared PEA HMAs.

by EOA. Addition of EOA as a replacement of EDA, replaced certain amide linkages by ester linkages. Ester linkages (formed by the reaction between EOA and lower purity dimer acid) were not able to form hydrogen bonding (intermolecular forces of attraction) as efficient as that formed by the amide linkage, due to the presence of $-\mathrm{H}$ atom in the primary amide linkage which was absent on the ester linkage. This led to the decrease in the crystallinity of the PEA HMA. Crystallinity of the HMA prepared using dimer acid, ethylenediamine, and EOA decreased with increase in concentration of EOA. Decrease in $\mathrm{Tg}, \mathrm{Tm}, \mathrm{Hm}, \mathrm{Tc}$, and Hc proves the occurrence of the above mentioned phenomena on addition of EOA, as those properties are directly proportional to crystallinity of the material. However, lower $\mathrm{Tg}$ indicates better low temperature flexibility for the HMAs prepared using dimer acid, ethylenediamine, and EOA. Low temperature flexibility would increase with increase in concentration of EOA in the PEA HMA.

4.5. Adhesion Properties. Adhesion properties like lap shear strength (LSS, MPa) and T-peel strength (TPS, N/mm) obtained for the prepared HMAs are listed in Table 6.

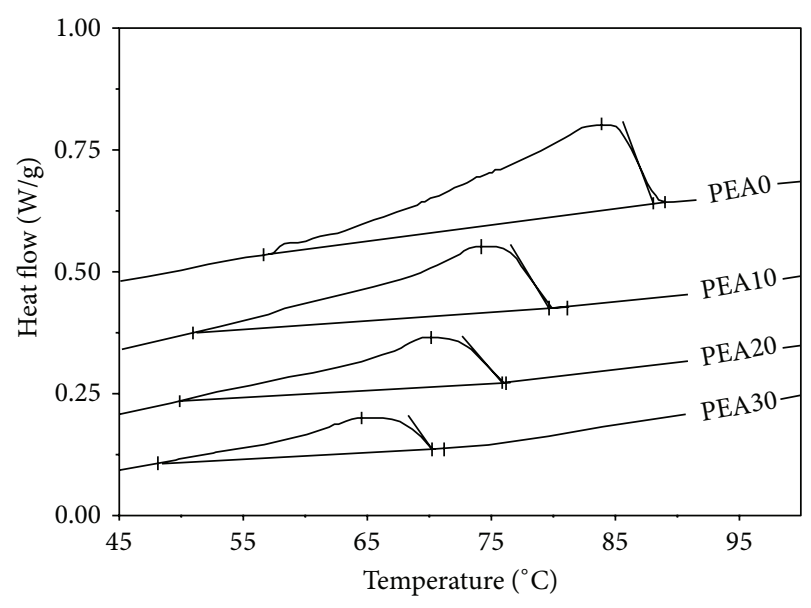

FIGURE 5: Cooling curves obtained for the prepared PEA HMAs.

TABLE 6: Adhesion properties obtained for the prepared PEA HMAs.

\begin{tabular}{lcc}
\hline Sample name & $\begin{array}{c}\text { Lap shear strength } \\
(\mathrm{MPa})\end{array}$ & $\begin{array}{c}\text { T-peel strength } \\
(\mathrm{N} / \mathrm{mm})\end{array}$ \\
\hline PEA0 & $7.1 \pm 0.4$ & $11.5 \pm 0.8$ \\
PEA10 & $6.5 \pm 0.3$ & $10.1 \pm 0.9$ \\
PEA20 & $5.1 \pm 0.5$ & $9.2 \pm 0.8$ \\
PEA30 & $4.4 \pm 0.4$ & $7.7 \pm 0.9$ \\
\hline
\end{tabular}

HMA prepared from lower purity dimer acid and EDA had been found to have LSS of 7.1 $\pm 0.4 \mathrm{MPa}$ and TPS of $11.5 \pm 0.8 \mathrm{~N} / \mathrm{mm}$. LSS and TPS decreased with increased replacement of EDA with EOA. As the concentration of EOA increased, intermolecular forces of attraction decreased, mainly due to the replacement of amide linkages by ester linkages. Decrease in intermolecular forces of attraction allowed the PEA molecules to flow past each other more easily on application of force in lap shear or T-peel joint, decreasing the adhesion strength of the prepared HMAs.

4.6. Rheological Properties. Figure 6 is a plot of viscosity ( $\mathrm{Pa}$. s) versus shear rate $\left(\mathrm{s}^{-1}\right)$ obtained for the prepared PEA HMAs at $200^{\circ} \mathrm{C}$. It can be seen that viscosity decreased with increase in shear rate. Thus, the prepared HMAs showed shear thinning behaviour. Also, the viscosity of the prepared PEA HMAs decreased with increased replacement of EDA by EOA, at same shear rate. Partial replacement of the amide linkage by the ester linkages decreased the hydrogen bond formation capacity of the HMA prepared using EOA. This led to the decrease in the intermolecular forces of attraction and thus the crystallinity of the HMA. Thus PEA HMAs containing higher quantity of EOA showed lower resistance to the rotating rheometer spindle, showing lower viscosity. These results strongly correlate with the observed mechanical and thermal properties.

Figure 7 is a plot of viscosity (Pa.s) versus time (s) obtained for the prepared PEA HMAs at $200^{\circ} \mathrm{C}$. It was determined to understand the effect of time on the viscosity 


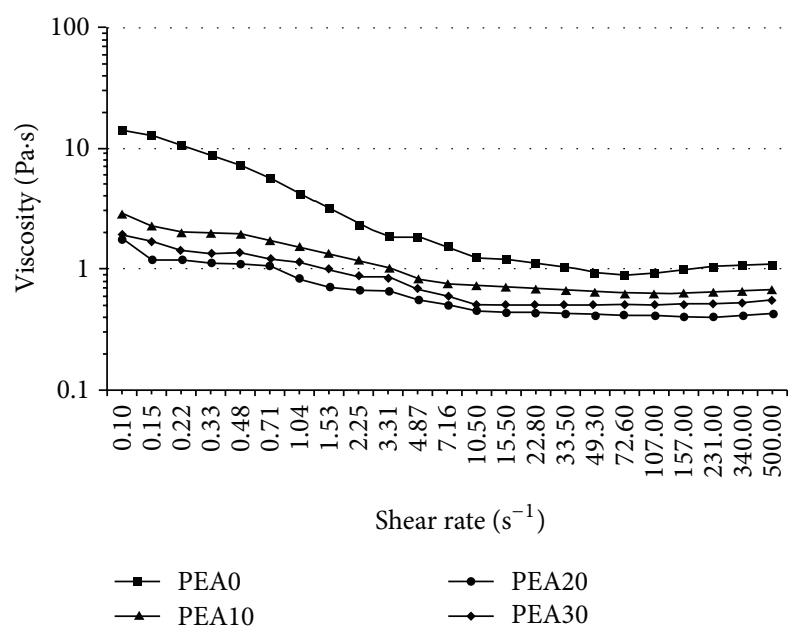

FIGURE 6: Plot of viscosity (Pa.s) versus shear rate $\left(\mathrm{s}^{-1}\right)$ obtained for the prepared PEA HMAs.

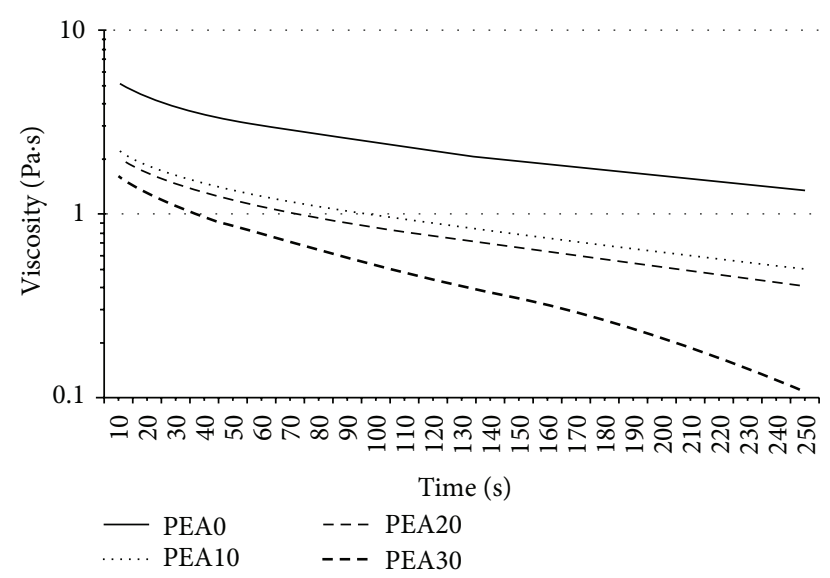

Figure 7: Plot of viscosity (Pa.s) versus time $\left(\mathrm{s}^{-1}\right)$ obtained for the prepared PEA HMAs.

of the prepared HMAs. In general, viscosity decreased with increase in time at constant temperature. Thus, the prepared HMAs showed thixotropic behaviour. Decrease in viscosities of PEA0, PEA10, and PEA20 with time was very less as compared to PEA30. Viscosity of PEA30 decreased drastically with time. This can be due to the decrease in crystallinity of HMA caused by EOA replacing EDA, which caused decrease in intermolecular forces of attraction. Intermolecular forces of attraction decreased due to the replacement of amide linkages by ester linkages.

\section{Conclusion}

Polyesteramide (PEA) based hot melt adhesives (HMAs) were successfully synthesized using ethanolamine (EOA) as a partial replacement of ethylenediamine (EDA) to react with the lower purity dimer acid. In the formulations, EDA was partially replaced by $10-30 \%$ (on molar basis) of EOA. Prepared PEA HMAs were characterized for mechanical, thermal, rheological, and adhesion properties. Crystallinity and thus the intermolecular forces of attraction played an important role in affecting the above said properties. It was found that tensile strength, melting temperature, enthalpy of melting, crystallization temperature, enthalpy of crystallization, glass transition temperature, lap shear strength, T-peel strength, and viscosity decreased with increased replacement of EDA by EOA. This was attributed to the diminished hydrogen bond formation capacity of ester linkage as compared to the amide linkage. However, lower glass transition temperature indicates better low temperature flexibility for the HMAs prepared using EOA and would also have better adhesion process due to lower viscosity and melting temperature.

\section{Conflict of Interests}

The authors do not have any direct financial relation with the commercial identities.

\section{Acknowledgment}

Authors would like to acknowledge the Institute of Chemical Technology (formerly UDCT) for permitting them to use the analytical facilities available at the institute.

\section{References}

[1] E. M. Yorkgitis, Adhesive Compounds-Encyclopedia of Polymer Science and Technology, John Wiley and Sons, New York, NY, USA, 2001.

[2] E. M. Petrie, Handbook of Adhesives and Sealants, McGraw Hill Professional, New York, NY, USA, 2000.

[3] S. Böhm, G. Hemken, E. Stammen, and K. Dilger, "Microbonding using hot melt adhesives," Journal of Adhesion and Interface, vol. 7, p. 28, 2006.

[4] C. R. Frihart, "Specific adhesion model for bonding hot-melt polyamides to vinyl," International Journal of Adhesion and Adhesives, vol. 24, no. 5, pp. 415-422, 2004.

[5] J. G. Martins and K. F. Ashley, "Poly(ester-amide) block copolymer hot melt adhesives," U.S. Patent 3,650,999, 1972.

[6] B. Krieger, M. Bolze, and M. Drawert, "Hot melt pressure sensitive adhesives," U.S. Patent 3,792,002, 1974.

[7] J. G. Martins and D. D. Donermeyer, "Cavity filling with poly(ester-amide) hot melt adhesive composition," U.S. Patent 4,073,973, 1978.

[8] J. G. Martins and D. D. Donermeyer, "Poly(ester-amide) hot melt adhesives containing spheroidal metal powders," U.S. Patent 4,097,445, 1978.

[9] R. L. Veazey, "Poly(ester-amide) hot-melt adhesives," U.S. Patent 4,485,233, 1984.

[10] C. R. Frihart and R. L. Veazey, "Poly(ester-amide) hot-melt adhesives," U.S. Patent 4,515,939, 1985.

[11] C. R. Frihart and R. L. Veazey, "Poly(ester-amide) hot-melt adhesives," U.S. Patent 4,569,985, 1986.

[12] C. R. Frihart and R. L. Veazey, "Poly(ester-amide) hot-melt adhesive," U.S. Patent 4,569,987, 1986.

[13] K. S. Hayes, C. R. Frihart, and R. J. Wroczynski, "Novel poly(ester-amide) hot-melt adhesives," U.S. Patent 4,611,051, 1986. 
[14] D. G. Swan and J. C. Hansen, "Poly(ester-amide) compositions," U.S. Patent 4,656,242, 1987.

[15] X. Chen, H. Zhong, L. Jia et al., "Polyesteramides used for hot melt adhesives: synthesis and effect of inherent viscosity on properties," Journal of Applied Polymer Science, vol. 82, no. 11, pp. 2696-2701, 2001.

[16] X.-M. Chen, H. Zhong, L.-Q. Jia, R.-G. Tang, J.-L. Qiao, and Z.Y. Zhang, "Effect of the content of EVA on the dispersing status and properties of polyesteramide/EVA blend," International Journal of Adhesion and Adhesives, vol. 21, no. 3, pp. 221-226, 2001.

[17] R. Broos, R. J. Kopmans, and R. Wevers, "Polyester-amide based hot melt adhesives," European Patent 1928969B1, 2010.

[18] E. Appleman, J. T. Carter, S. Van, and J. G. Renee, "Polyesteramide copolymer," European Patent 1397415B1, 2010.

[19] P. G. Kadam, R. A. Kute, and S. T. Mhaske, "Synthesis and characterization of a novel polyesteramide from modified jatropha seed oil, dimer acid and ethylenediamine as hot melt adhesive," Journal of Adhesion Science and Technology, vol. 28, no. 7, pp. 675-689, 2013. 

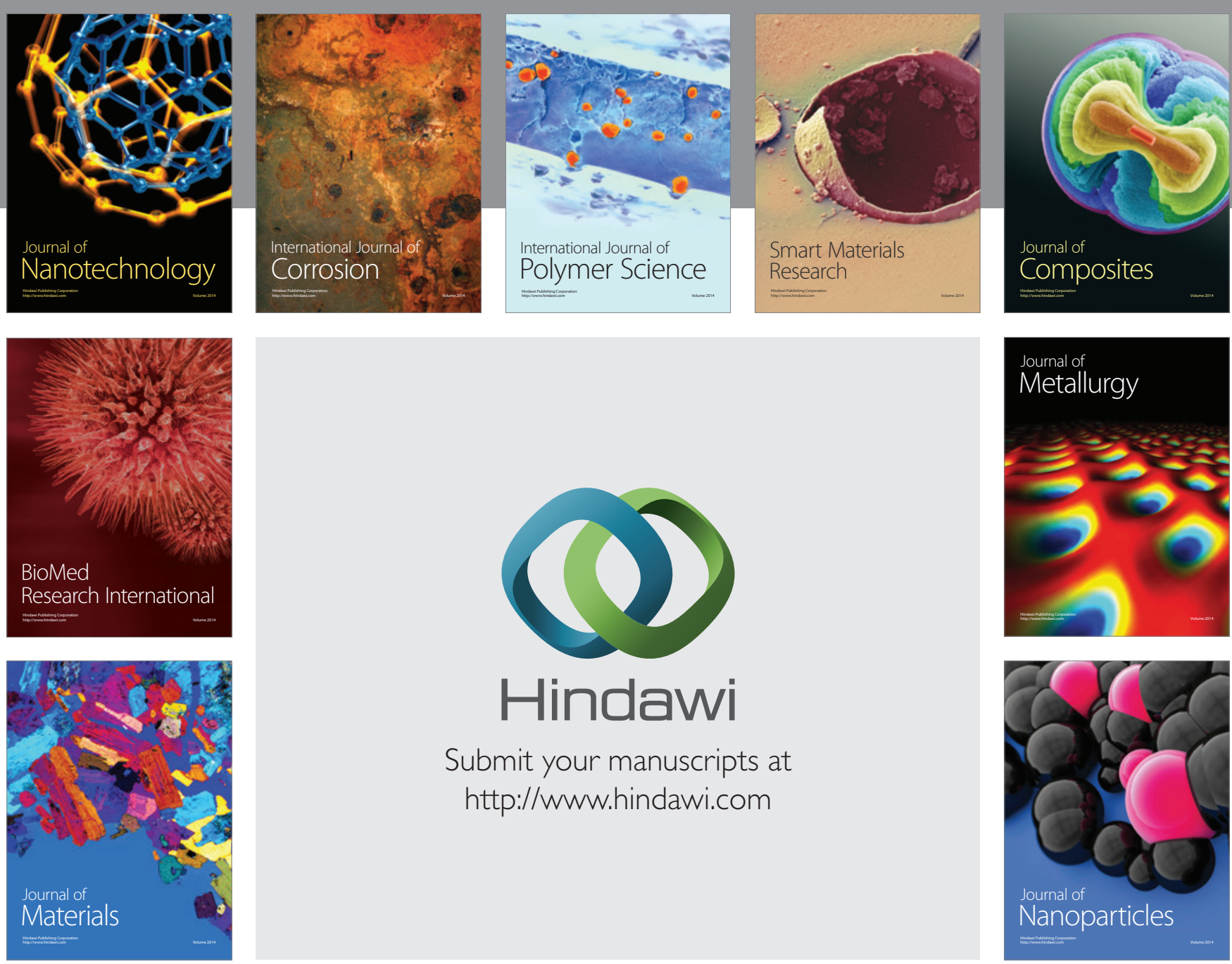

Submit your manuscripts at http://www.hindawi.com
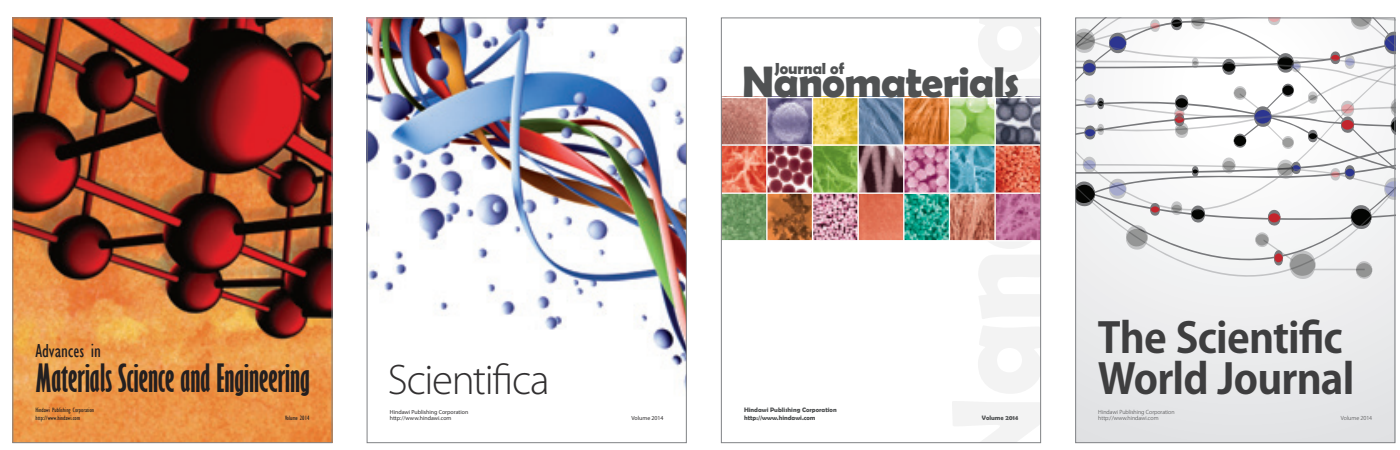

\section{The Scientific World Journal}
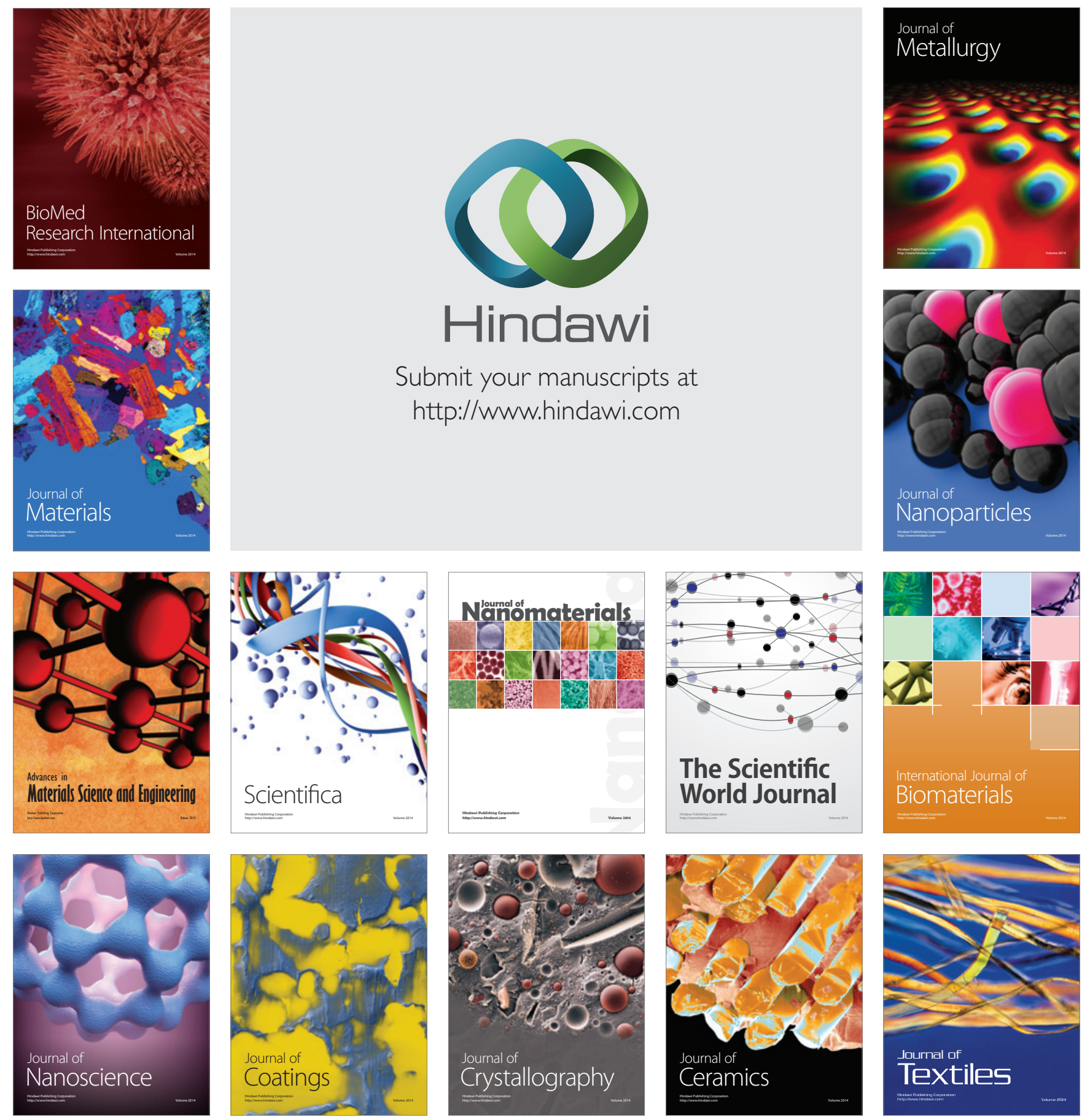